\title{
Low-loss SiN waveguides for efficient and broadly tunable nonlinear frequency conversion
}

\author{
Florent Mazeas $^{1 *}$, Junqiu Liu², Tobias J. Kippenberg ${ }^{2}$, Camille-Sophie Brès ${ }^{1 *}$ \\ ${ }^{1}$ Ecole Polytechnique Fédérale de Lausanne, Photonic Systems Laboratory (PHOSL), STI-IEL, Station 11, CH-1015 Lausanne, Switzerland \\ ${ }^{2}$ Ecole Polytechnique Fédérale de Lausanne, Laboratory of Photonics and Quantum Measurements (LPQM), SB-IPHYS, Station 3, CH-1015 \\ Lausanne, Switzerland \\ *florent.mazeas.epfl.ch, camille.Bres@epfl.ch
}

\begin{abstract}
We report on the use of low-loss $\mathrm{Si}_{3} \mathrm{~N}_{4}$ waveguides for efficient frequency conversion tunable from the near infrared to the short-wave infrared. We achieve $-15 \mathrm{~dB}$ conversion efficiency for $200 \mathrm{~mW}$ coupled pump power. @ 2020 The Author(s)
\end{abstract}

\section{Introduction}

Nowadays, integrated photonics structures are widely used both for their linear and nonlinear properties. Owing to high optical confinement and strong intrinsic nonlinear material properties, we can efficiently exploit nonlinear phenomena while keeping the size of the device at the chip level. Several platforms appear suitable for nonlinear integrated optics such as silicon $(\mathrm{Si})$, silica $\left(\mathrm{SiO}_{2}\right)$, silicon nitride $\left(\mathrm{Si}_{3} \mathrm{~N}_{4}\right)$, chalcogenide glasses and III-V semiconductors [1]. They all present their specific advantages and drawbacks. While silicon and chalcogenides offer some of the highest intrinsic nonlinearity, the former suffers from high nonlinear losses at telecommunication wavelengths while the latter is not CMOS fabrication compatible and still lacks reliability. Silica has weak nonlinearity and starts strongly absorbing beyond 2 microns. Regarding III-V approaches, high performance devices such as low-loss dispersion engineered waveguides are still difficult to realize. Therefore, $\mathrm{Si}_{3} \mathrm{~N}_{4}$ appears to be a good compromise thanks to its CMOS fabrication compatibility, low linear losses, relatively high nonlinearity, and larger bandgap compared to silicon which allows operation from the visible to the middle infrared with very low two-photons absorption and free carrier absorption. A specifically challenging task is still however the realization of broadband and efficient continuous wave (CW) four-wave mixing (FWM) in CMOS fabrication compatible waveguides. This comes from the trade-off between bandwidth and efficiency when pump power is limited, as is often the case in CW regime: while efficiency can be improved by increasing the interaction length, it leads to a reduction of the bandwidth for a constant pump power. In such platforms, dispersion optimization also becomes particularly critical.

Here we show efficient FWM with CW pumping in low-loss $\mathrm{Si}_{3} \mathrm{~N}_{4}$ waveguides, reaching $-15 \mathrm{~dB}$ with $200 \mathrm{~mW}$ pump. Operation at both $1550 \mathrm{~nm}$ and $2000 \mathrm{~nm}$ is possible, with $30 \mathrm{~nm}$ and $60 \mathrm{~nm} 3 \mathrm{~dB}$ bandwidth, respectively, despite the use of $\mathrm{cm}$-long waveguides. The waveguides have low loss in combination of large cross sections, which allows for dispersion engineering.

2. Experiments and results
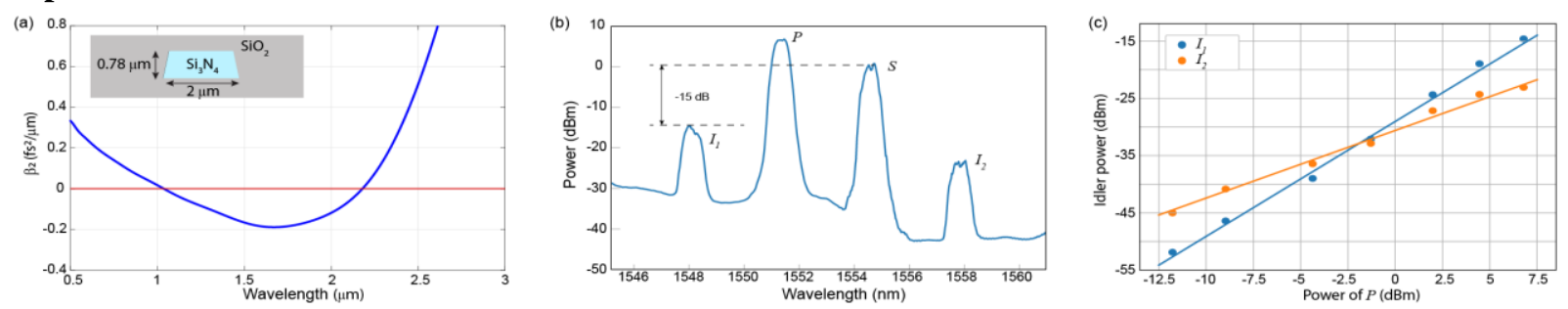

Figure 1. (a) Theoretical dispersion of the $2 \times 0.78 \mu \mathrm{m}^{2}$ waveguide. Inset schematic of the waveguide cross-section (b) Spectrum at the output of the $32.2 \mathrm{~cm}$ waveguide for $200 \mathrm{~mW}$ of coupled pump power. (c) Idler power $\left(I_{I}\right.$ and $\left.I_{2}\right)$ as a function of the power of $P$ for constant signal power. We have a quadratic and linear dependence, respectively.

The waveguides used in this study have a cross section of $2.0 \times 0.78 \mu \mathrm{m}^{2}$. Their estimated propagation loss is around $0.05 \mathrm{~dB} / \mathrm{cm}$ allowing for the design of long waveguides. The waveguides are folded in spirals and we tested two different lengths of $16.5 \mathrm{~cm}$ and $32.2 \mathrm{~cm}$. The dispersion of the waveguide is tailored as to provide a relatively low and flat anomalous dispersion between 1 and $2.2 \mu \mathrm{m}$ as seen in Fig.1(a). Combined with the enhanced transparency window, this design is suitable for FWM process in the telecom and short-wave infrared 
simultaneously as opposed to previous work [2,3]. The experimental pump-probe setup operates both at 1.5 and 2 $\mu \mathrm{m}$. For the former, we couple two tunable amplified sources through a wavelength-division multiplexer. For the later, a fixed laser diode at $2004 \mathrm{~nm}$ is amplified and serves as the pump while a home-made Thulium doped fiber laser tunable from $1800 \mathrm{~nm}$ to $2000 \mathrm{~nm}$ serves as the signal. On both cases, light is injected into the waveguides using micro-lensed fibers with an estimated coupling loss around $8 \mathrm{~dB}$ per facet.

Fig. 1(b) shows the spectrum at the output of the $32.2 \mathrm{~cm}$ waveguide for $200 \mathrm{~mW}$ of pump power coupled $(P)$ together with a signal (S) both in the telecom band. We measure $-15 \mathrm{~dB}$ conversion efficiency (CE), which is, to the best of our knowledge, the highest value reported for $\mathrm{CW}$-pumped frequency conversion in a non-resonant $\mathrm{Si}_{3} \mathrm{~N}_{4}$ waveguide. We varied power of $P$ keeping the power of $S$ constant as to measure the growth dependence of idler $I_{l}$ and idler $I_{2}$ (Fig. 1 (c)). As expected we observe a quadratic dependence for the CE to $I_{l}$ (since $P$ acts as the pump) and linear for $I_{2}$ (since $P$ acts as a signal).
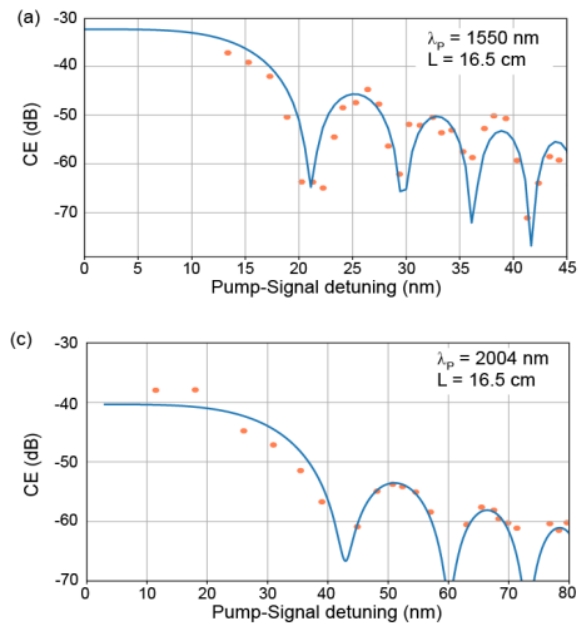
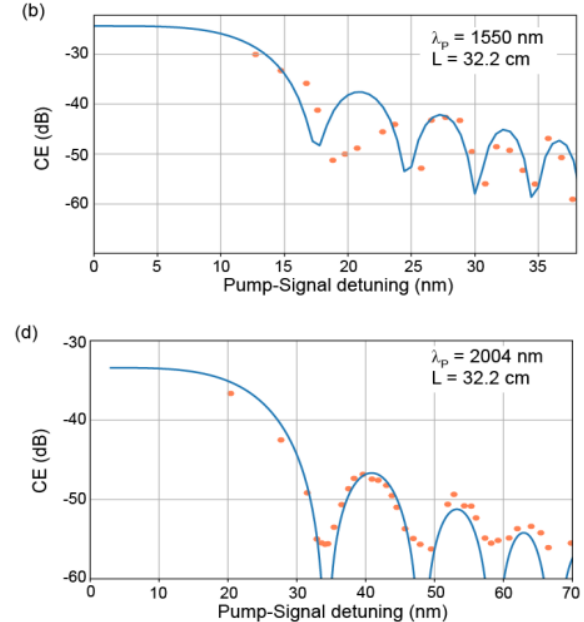

Figure 2: CE curves as a function of pump-signal detuning for (a) pump at $1550 \mathrm{~nm}$ in a $16.5 \mathrm{~cm}$ long waveguide, (b) pump at $1550 \mathrm{~nm}$ in a $32.2 \mathrm{~cm}$ long waveguide, (c) pump at $2004 \mathrm{~nm}$ in a $16.4 \mathrm{~cm}$ long waveguide and (d) pump at $2004 \mathrm{~nm}$ in a 32.2 $\mathrm{cm}$ long waveguide. Points: experimental data; Line: theoretical fit.

In Fig.2, we plot the CE as a function of the pump-signal detuning. The results for the telecom band are shown in Fig.2 (a,b), for the waveguide of length $32.2 \mathrm{~cm}$ and $16.5 \mathrm{~cm}$, respectively. We estimate the pump power near $100 \mathrm{~mW}$. Similarly, the results for the 2.04 micron pump are in Fig. 2(c,d) and a pump power of approximately 79 $\mathrm{mW}$. Based on measurements obtained with a signal located a few $\mathrm{nm}$ from the pump, we estimate the nonlinear coefficient $\gamma=0.62 \mathrm{~W}^{-1} \mathrm{~m}^{-1}$, in agreement with theoretically predicted value. We note that as expected, the CE decreased by close to $6 \mathrm{~dB}$ in the shorter waveguides (half the length) while the bandwidth increased. By fitting the experimental data, we can extract the expected dispersion at the pump wavelength. We observe that the $3 \mathrm{~dB}$ bandwidth is wider in the 2 microns band, going from approximately $30 \mathrm{~nm}$ to $60 \mathrm{~nm}$ (two sided), an indication of a lower dispersion value. The extracted dispersion values $\beta_{2 \_1550 \mathrm{~nm}}=-0.304 \pm 0.044 \mathrm{fs}^{2} / \mu \mathrm{m}$ at $1550 \mathrm{~nm}$ and $\beta_{2 \_2004 \mathrm{~nm}}=-0.206 \pm 0.02 \mathrm{fs}^{2} \backslash \mu \mathrm{m}$ at $2004 \mathrm{~nm}$. While the extracted values are slightly higher than the expected ones (see Fig. 1(a)), the trend is similar and we estimate the zero-dispersion-wavelength to be between 2.2 and $2.3 \mu \mathrm{m}$.

To conclude, we show continuous-wave conversion efficiency of $-15 \mathrm{~dB}$ with $200 \mathrm{~mW}$ of telecom pump power and $-35 \mathrm{~dB}$ with $79 \mathrm{~mW}$ at 2 microns, over a large bandwidth. Owing to the low loss features of our $\mathrm{Si}_{3} \mathrm{~N}_{4}$ waveguides, the $\mathrm{CE}$ could be further increased by using longer waveguides. In addition, our waveguides have a large cross section, which allows for further dispersion engineering, which was not possible in other low loss waveguides [2]. Flattening of the dispersion would enable wider bandwidth without sacrificing efficiency.

[1] M. Pu, H. Hu, L. Ottaviano, E. Semenova, D. Vukovic, L.K Oxenløwe, K. Yvind, Ultra-Efficient and Broadband Nonlinear AlGaAs-on-Insulator Chip for Low-Power Optical Signal Processing, Laser \& Photonics Reviews, 12, 1800111, 2018

[2] C. J. Kruckel, V. Torres-Company, P.A. Andrekson, D.T. Spencer, J.F. Bauters, M.J.R. Heck, J. E. Bowers, Continuous wave-pumped wavelength conversion in low-loss silicon nitride waveguides, Optics Letters, Vol. 40, No. 6, 2015,

[3] M. Lamy, C. Finot, P. Colman, J. Fatome, G. Millot, G. Roelkens, B. Kuyken, K. Hammani, Silicon Waveguides for HighSpeed Optical Transmissions and Parametric Conversion Around $2 \mu \mathrm{m}$, IEEE Photonics Technol. Lett. , 31, 165-168, 2019 